\title{
Article
}

\section{A systematic review of community pharmacies' staff diagnostic assessment and performance in patient consultations}

Sinopoulou, Vassiliki, Gordon, Morris and Rutter, Paul

Available at http://clok.uclan.ac.uk/24452/

Sinopoulou, Vassiliki ORCID: 0000-0002-2831-9406, Gordon, Morris ORCID: 0000-0002-1216-5158 and Rutter, Paul ORCID: 0000-0003-4106-1515 (2019) A systematic review of community pharmacies' staff diagnostic assessment and performance in patient consultations. Research in Social and Administrative Pharmacy, 15 (9). pp. 1068-1079. ISSN 1551-7411

It is advisable to refer to the publisher's version if you intend to cite from the work. http://dx.doi.org/10.1016/j.sapharm.2018.10.003

For more information about UCLan's research in this area go to http://www.uclan.ac.uk/researchgroups/ and search for <name of research Group>.

For information about Research generally at UCLan please go to http://www.uclan.ac.uk/research/

All outputs in CLoK are protected by Intellectual Property Rights law, including Copyright law. Copyright, IPR and Moral Rights for the works on this site are retained by the individual authors and/or other copyright owners. Terms and conditions for use of this material are defined in the policies page. 


\title{
Manuscript Details
}

\section{Manuscript number}

Title

Article type
RSAP_2018_202_R2

A systematic review of community pharmacies' staff diagnostic assessment and performance in patient consultations

Review Article

\begin{abstract}
Background: Increases in patients seeking advice at pharmacies has led to pharmacy staff engaging in diagnostic behaviours. Approaches to diagnosis include using mnemonics and clinical reasoning. Objectives: The primary aim of this review was to assess the degree to which the criteria authors use to evaluate diagnostic performance in pharmacy consultations, in studies that use simulated patients or vignettes, conform with a clinical reasoning and a mnemonic framework. A secondary aim of the review was to characterise staff performance in the studies, based on the authors' comments of their results. Methods: MEDLINE, EMBASE and Web of Science were searched between October 2016 and April 2017. Only peer-reviewed studies assessing pharmacy staff's diagnostic performance by using simulated patients or vignettes were eligible for inclusion. Data were extracted about how each study's criteria conformed with clinical reasoning and mnemonic frameworks. A scoring system between 0 and 4 was devised to determine the degree to which studies aligned to these two approaches. Risk of bias was assessed using the NHI Study Quality Assessment Tools. The review was registered in PROSPERO with identification number CRD42017054827. Results: Sixty-eight studies (55 cross-sectional, 11 educational interventions and 2 RCTs) with sample sizes between 10 and 2700 were included in the review. Most studies were of poor or fair quality. Performance of pharmacy staff was overwhelmingly reported as poor by study authors. This was the case regardless of geography, scenario used or whichever assessment framework was utilised. Scrutiny on how authors arrived at these conclusions revealed that mnemonic criteria were employed to assess pharmacy staff's diagnostic performance rather than a clinical reasoning approach. Conclusions: Potentially important aspects of the decision-making process, that clinical reasoning accounts for, were left unexplored. The scope of the number and locations of the included studies is a strength of this review, however, the system employed does not represent a validated tool.
\end{abstract}

Keywords

Manuscript category

Corresponding Author

Corresponding Author's Institution

Order of Authors community pharmacy; diagnosis; clinical reasoning; mnemonics;

Research Paper

Vassiliki Sinopoulou

NESLI University of Central Lancashire

Vassiliki Sinopoulou, Morris Gordon, Paul Rutter

\section{Submission Files Included in this PDF}

\author{
File Name [File Type] \\ cover letter.docx [Cover Letter]
}

Response to reviewers.docx [Response to Reviewers (without Author Details)]

\author{
Abstract.docx [Abstract] \\ Title page with author details.docx [Title Page (with Author Details)] \\ re-revised submission.docx [Manuscript (without Author Details)]
}

To view all the submission files, including those not included in the PDF, click on the manuscript title on your EVISE Homepage, then click 'Download zip file'. 


\section{Research Data Related to this Submission}

Data set

https://data.mendeley.com/datasets/n2h8t6vg54/draft?a=b2ab23c1-73bd-411aaea5-83a4598a2331

Data for: A systematic review of community pharmacies' staff diagnostic assessment and performance in patient consultations

The dataset includes reference list of the included studies, the extracted data for both extraction rounds and the analysis of the results presented in the paper. 
COVER LETTER FOR SUBMISSION OF MANUSCRIPT

Subject: SUBMISSION OF A MANUSCRIPT FOR EVALUATION

Dear Editor,

I submit herewith a manuscript entitled "A systematic review of community pharmacies' staff diagnostic assessment and performance in patient consultations" for publication in "Research in Social and Administrative Pharmacy" for possible evaluation.

With the submission of this manuscript all authors give consent for its publication and would like to undertake that the above mentioned manuscript has not been published elsewhere or is being considered for publication elsewhere.

Thank you.

Sincerely,

Vassiliki Sinopoulou

School of Pharmacy and Biomedical Sciences

University of Central Lancashire

vsinopoulou@uclan.ac.uk 


\section{Response to reviewers:}

Once more, we would like to thank all reviewers for their comments and we greatly appreciate the time they put in reviewing our paper. We were very happy to read that our paper would be accepted for publication, pending these minor changes. As previously, we have tried to respond to each comment as it was presented to us. The original comments appear in italics and our responses are in plain text below each comment. Hopefully any changes can be easily traced based, as line count was not greatly affected.

\section{Reviewer 1}

Thank you for the opportunity to review this revised manuscript. I am happy to confirm that overall the authors have addressed the issues raised in my review. In a number of instances the responses illustrate some limitations of the methodology e.g. it would have been preferable for the additional databases explored to have been formally included in the PRISMA flowchart showing the larger number of replicates arising from these additional searches which were then excluded, rather than only addressed informally; similarly it would have been preferable to have retained a manual record of inter-rater agreement statistics as this is not captured by the Covidence software. However, as these data are unavailable, the manner in which the authors have addressed the issues in the manuscript is acceptable. A small number of typographical/grammatical errors and inconsistencies remain (including some in new sections such as the conclusion and the amended tables, which it should be possible to remove in final proofreading. However, overall the manuscript describes a worthwhile study which is likely to be of interest to a broad readership, and I recommend its publication.

We are very happy to hear this reviewer is satisfied with our responses and in any future work we will make sure that the database issue and inter-rater agreement are addressed better. We have tried to correct any remaining typographical/grammatical errors and inconsistencies but as these were not explicitly identified we might have missed some, especially since after reading the manuscript so many times we might have become blind to them. We apologise for this and we hope that they might be picked up by an editor's "fresh pair of eyes" before publication.

\section{Reviewer 4}

Noted the addressed comments to previous reviewers.

Minor comments which can be considered but do not change the content of the article. No changes would preclude me from accepting the article.

1. Line 93, why is nursing in parentheses? this seems to entail a judgement that I am unsure you wanted to make. 
We have removed the parentheses.

2. Line 165 , knowing what they scenario... you mean the scenario

We have made the correction.

3. Lines 182 - 189, this lacked clarity. I think this is in response to previous reviewer to explain why you didn't use additional databases so may need to keep it but could be clearer if written in shorter, active tense

We have rephrased this in an effort to be more clear.

4 Lack a reference for line 366, UN country classification

We have provided a link to the UN document.

4. Line 396 , as this studies... you mean these studies

We made the correction.

5. Line 464, you lack a reference for WWHAM. You should spell this out for international audiences prior to using

We have spelled it out.

7. Line 502, A better transition between the scoring and the mnemonic would help your reader. It is an abrupt transition

We were not entirely sure what the "transition between the scoring and the mnemonic" refers to but we have rephrased line 502 to hopefully make it more clear.

8. Line 519 - 520, The use of the words "few" and "some" lead your reader to judgement. Implicitly few seems like less articles than some to me, as a reader. Consider using specific numerical values instead of putting those in parentheses

We have removed the use of "few" and "some" and replaced them with the actual numbers.

9. Under section "study authors' outlook on performance" - you could provide examples of what a positive and negative outlook were to help the audience 
understand what you classified each way. Consider reviewing examples of "quotes for qualitative interviews"

We have provided one positive and one negative example. However, we are unsure about what was meant by "Consider reviewing examples of 'quotes for qualitative interviews'".

10. Line 553, you discuss that overwhelmingly reported as poor, but if the framework ratings are poor (e.g. "0") can you use them to provide you a valid response.

The "overwhelmingly reported as poor" quote refers to the authors' outlook on performance in their studies, not our own. The framework ratings we used were to scrutinise what methods authors used to arrive to their conclusions. We would need further clarification of this comment to understand it better, if our response is not enough.

11. Line 604, should only new tools be validated or should previous tools be validated? Your framework did not show positive

We have removed the word "new" to indicate that any tools should be validated, whether entirely new or validated versions of older ones. The last sentence of the comment appears to be unfinished and we are not unsure about what the reviewer meant to say.

12. Line 669, relies on mnemonic than a clinical, should be mnemonic rather than a clinical

We have corrected this. 


\section{Abstract}

Background: Increases in patients seeking advice at pharmacies has led to pharmacy staff engaging in diagnostic behaviours. Approaches to diagnosis include using mnemonics and clinical reasoning.

Objectives: The primary aim of this review was to assess the degree to which the criteria authors use to evaluate diagnostic performance in pharmacy consultations, in studies that use simulated patients or vignettes, conform with a clinical reasoning and a mnemonic framework. A secondary aim of the review was to characterise staff performance in the studies, based on the authors' comments of their results.

Methods: MEDLINE, EMBASE and Web of Science were searched between October 2016 and April 2017. Only peer-reviewed studies assessing pharmacy staff's diagnostic performance by using simulated patients or vignettes were eligible for inclusion. Data were extracted about how each study's criteria conformed with clinical reasoning and mnemonic frameworks. A scoring system between 0 and 4 was devised to determine the degree to which studies aligned to these two approaches. Risk of bias was assessed using the NHI Study Quality Assessment Tools. The review was registered in PROSPERO with identification number CRD42017054827.

Results: Sixty-eight studies (55 cross-sectional, 11 educational interventions and 2 RCTs) with sample sizes between 10 and 2700 were included in the review. Most studies were of poor or fair quality. Performance of pharmacy staff was overwhelmingly reported as poor by study authors. This was the case regardless of geography, scenario used or whichever assessment framework was utilised. Scrutiny on how authors arrived at these conclusions revealed that mnemonic criteria were employed to assess pharmacy staff's diagnostic performance rather than a clinical reasoning approach.

Conclusions: Potentially important aspects of the decision-making process, that clinical reasoning accounts for, were left unexplored. The scope of the number and locations of the included studies is a strength of this review, however, the system employed does not represent a validated tool. 
A systematic review of community pharmacies' staff diagnostic assessment and performance in patient consultations

Vassiliki Sinopoulou, School of Pharmacy and Biomedical Sciences, University of Central Lancashire, email: VSinopoulou@uclan.ac.uk

Dr. Morris Gordon, School of Medicine, University of Central Lancashire

Professor Paul Rutter, School of Pharmacy and Biomedical Sciences, University of Central Lancashire 


\section{A systematic review of community pharmacies' staff diagnostic assessment and performance in patient consultations}

Background: Increases in patients seeking advice at pharmacies has led to pharmacy staff engaging in diagnostic behaviours. Approaches to diagnosis include using mnemonics and clinical reasoning.

Objectives: The primary aim of this review was to assess the degree to which the criteria authors use to evaluate diagnostic performance in pharmacy consultations, in studies that use simulated patients or vignettes, conform with a clinical reasoning and a mnemonic framework. A secondary aim of the review was to characterise staff performance in the studies, based on the authors' comments of their results.

Methods: MEDLINE, EMBASE and Web of Science were searched between October 2016 and April 2017. Only peer-reviewed studies assessing pharmacy staff's diagnostic performance by using simulated patients or vignettes were eligible for inclusion. Data were extracted about how each study's criteria conformed with clinical reasoning and mnemonic frameworks. A scoring system between 0 and 4 was devised to determine the degree to which studies aligned to these two approaches. Risk of bias was assessed using the NHI Study Quality Assessment Tools. The review was registered in PROSPERO with identification number CRD42017054827.

Results: Sixty-eight studies (55 cross-sectional, 11 educational interventions and 2 RCTs) with sample sizes between 10 and 2700 were included in the review. Most studies were of poor or fair quality. Performance of pharmacy staff was overwhelmingly reported as poor by study authors. This was the case regardless of geography, scenario used or whichever assessment framework was utilised. Scrutiny on how authors arrived at these conclusions revealed that mnemonic criteria were employed to assess pharmacy staff's diagnostic performance rather than a clinical reasoning approach.

Conclusions: Potentially important aspects of the decision-making process, that clinical reasoning accounts for, were left unexplored. The scope of the number and locations of the included studies is a strength of this review, however, the system employed does not represent a validated tool. 


\section{Introduction}

In recent decades self-care has been heavily promoted worldwide due to rising health-care $\operatorname{costs}^{1,2}$. This move toward patient empowerment has been supported by an increase in medicines being available to the public without the need for a prescription ${ }^{3}$. Community pharmacy staff are ideally placed to facilitate patient self-care and self-medication ${ }^{4-6}$ and indeed, community pharmacies have seen a rise in patients who visit in order to ask for help on minor ailments and advice on appropriate medication7. However, community pharmacists tend to find accommodating this task particularly challenging ${ }^{7}$ due to time constraints and therefore most consultations are often first conducted by counter staff who do not possess the knowledge and experience of pharmacists ${ }^{8,9}$.

To support pharmacy staff in this role, various protocols and guidelines, often using mnemonics, have been widely advocated and adopted, as they are easy to remember and quick to implement ${ }^{10}$. This, in theory, allows standardised questions to be asked, which are the same in every consultation and will help gather all the necessary information for a diagnosis and an appropriate action to be taken. However, data suggests that these standardised methods do not necessarily improve consultation performance ${ }^{11-13}$, possibly because staff may ask questions with no direct relation to the examined conditions and the gathered information is not useful for the decision-making process.

In medicine and nursing clinical reasoning processes are extensively used ${ }^{14}$. Clinical reasoning is an evidence-based, dynamic process in which the health professional combines scientific knowledge, clinical experience and critical thinking, with existing and newly gathered information about the patient. By the end of the process, all available information and logical inferences lead to the formation of a diagnosis ${ }^{15-17}$. This method has the disadvantage of being difficult to describe and hard to learn but it has the major advantage of improving clinical ability and being an effective method in establishing a diagnosis, possibly because all consultation information, either gathered through questioning or examining the patient, plays a part in the decision-making. In a community pharmacy context reaching decisions is equally pertinent but appears to be poorly described in the literature.

A previous review ${ }^{18}$, examined the rate and type of information gathered during community pharmacy consultations (only in developing economies) based on "common themes of the types of information that should be included in the information gathering process according to the literature". Besides examining the rate and type of gathered information, however, it 
is also important to examine their relevance and purpose and how they contribute to the decision-making process. It is not known if and to what degree these aspects of a consultation, which are related to clinical-reasoning, are performed in pharmacy settings. .

The primary aim of this review was to assess the degree to which the criteria authors use to evaluate diagnostic performance in pharmacy consultations, in studies that use simulated patients or vignettes, conform with a clinical reasoning and a mnemonic framework. A secondary aim of the review was to characterise staff performance in the studies, based on the authors' comments of their results.

\section{Methods}

A protocol for the review was submitted to PROSPERO with identification number CRD42017054827 and can be accessed at: http://www.crd.york.ac.uk/PROSPERO/display_record.php?ID=CRD42017054827.

The inclusion criteria were that the study assessed pharmacy staff diagnostic performance and the assessment was described in some form; the pharmacist/pharmacy staff should have been presented with and required to have responded to a diagnostic scenario; the scenario was presented in the form of simulated patients (SPs) or vignettes. Any study design was considered, including cross-sectional studies, interventions and randomised controlled trials. Studies needed to be peer-reviewed and published in English; no limit was set on publication date.

Studies were excluded when they did not provide an assessment of performance or a description of the assessment; the pharmacist/staff had to deal with an already diagnosed condition; they only looked at pharmacists'/staff's opinions on performance; they asked pharmacists/staff to follow specific screening methods or pre-set guidance that would prevent the potential use of any critical thinking.

The rationale behind the eligibility criteria was to include any study that had pharmacists and pharmacy staff presented with a diagnostic scenario to which they had to respond without knowing what the scenario would be and in a setting as close to a real life consultation as possible. A more exhaustive list of inclusion/exclusion criteria was included in the PROSPERO protocol, however, only the criteria that were encountered during the selection process are mentioned above.

Databases used to identify eligible studies were MEDLINE, EMBASE and Web of Science. Two of the authors (VS and PR) performed an initial scoping of the literature using the search 
algorithm ("community pharmac*") AND ("simulated patients" OR "mystery shopp*" OR "secret shopp*" OR "pseudo*" OR vignette*). Originally, we planned to adapt the algorithm further, however, more detailed iterations of the algorithm did not improve the search results and we eventually decided not to change it. Use of other bibliographic databases, as mentioned in the PROSPERO protocol, only provided us with duplicate results to the three databases previously mentioned and thus we decided not to use more databases.

Two rounds of searches were carried out. The first one took place on 03-11-2016 and the last one on10-4-2017, immediately before the extraction process. During the second round of searches, the reference lists of related literature were searched for additional titles. VS and PR independently screened the retrieved results titles and abstracts with the third reviewer (MG) acting as arbitrator whenever conflicts arose and the same process was followed for full text screening. The website platform Covidence was used to facilitate this process.

Risk of bias analysis was conducted at study level for each included study using the National Blood, Heart and Lung Institute's (NIH) of the U.S. Department of Health and Human Services Study Assessment Quality Tools, which rate studies as "good", "fair" or "poor". The Quality Assessment Tool for Observational Cohort and Cross-Sectional Studies was used for the included cross-sectional studies and the Quality Assessment of Controlled Intervention Studies tool was used for the included educational interventions and randomised controlled trials (RCTs). The analysis was conducted by VS, PR reviewed it and disagreements were resolved through discussion.

Data were manually extracted and inserted in to Microsoft Excel by VS and checked for accuracy by PR. The extracted data were; study characteristics: publication year, country, type of study, participant characteristics, type of results (quantitative or qualitative); quality characteristics: reporting of study piloting, SP training, data collection method ; methodology characteristics: type of methodology (SPs or vignettes), number of SPs, number of scenarios, type of scenarios (symptom presentation or product request), SP role (presenting for themselves or someone else); assessment characteristics: how assessment criteria were derived, whether studies assessed diagnostic performance in general or focused on the diagnosis of a specific condition, which medical conditions were used for the scenarios, whether staff knowledge was assessed and whether studies compared community pharmacists' performance with other pharmacy staff performance. A sub-analysis was conducted to establish whether there were differences in the types of conditions that studies 
of developed and developing economies focused on as this might have been a reason for subsequent differences in results between countries of different economic profiles.

Descriptive statistics (total numbers and percentages) were used to report the results.

Data were also extracted about how each study's criteria conformed with clinical reasoning and mnemonic frameworks. To achieve this, both were broken down into 4 characteristics (see below) and study texts were qualitatively analysed and coded for passages that corresponded to each characteristic. A value of one was assigned for each characteristic exhibited, meaning each study could score between 0 and 4. Mode and modal values were used to report the results.

These characteristics were developed by VS, after an initial familiarisation with the included studies, as summaries of key aspects of clinical reasoning and mnemonic questioning that the authors could have reflected on in their studies. The characteristics were reviewed by PR and, after discussion, adjustments were made until an agreement was reached. All included studies were then searched by VS for passages that indicated whether the authors have considered each respective characteristic in the methodology they used, when reporting their results or when discussing their results. If that had occurred the study would be awarded one point per characteristic, if not it would be awarded no points. The scoring was reviewed by PR and disagreements were resolved through discussion.

The characteristics for each framework were the following.

For the clinical reasoning framework:

1. The authors assessed staff against questions with specific relevance to the scenario condition (this is to show a basic level of clinical reasoning, even if they don't explicitly mention how or why the questions are relevant) e.g. for an emergency contraception scenario staff were expected to ask the question "when was your last menstrual cycle"

2. The authors have mentioned the purpose of the questions they assessed staff against (this shows that authors have considered why the questions are asked) e.g. for a sleeplessness scenario, patients' were asked about their medication because it might be causing or contributing to the patient's sleeplessness

3. The authors have reflected on how staff use the gathered information during the decision-making process (this is to show that authors have considered the importance of information interpretation in some way) e.g. in a dyspepsia scenario, a response to 
the question about pain location led the pharmacist to consider indigestion as a possibility

4. The authors considered whether there is a connection between the information gathered and the final decision taken by staff (to show that information gathering should be used for decision-making, even if the decision-making process is not described)

For the mnemonic framework:

1. The authors have assessed staff against questions (regardless of whether they're relevant to the condition or not) e.g. in a common cold scenario staff were expected to ask the patient's age

2. The authors have assessed staff against a checklist of questions they were expected to ask e.g. $\bullet$ Check symptoms $\bullet$ Check length of symptoms $\bullet$ Check other medication - Check other health condition $\bullet$ Refer if needed $\bullet$ Provide information

3. The authors have explicitly mentioned they used a known mnemonic method, guidelines or recommendations to assess performance e.g. WWHAM, WHO guidelines, Australian practice? recommendations

4. The authors have reported the final decision staff took (irrespective of whether it was connected to the information gathering or not) e.g. "In $90 \%$ of the scenarios not appropriate for self- medication, a recommendation was made for the customer to see a physician/GP, but in only $30 \%$ of those referrals was there sufficient urgency"

Each study was also coded for passages that indicated whether the authors' outlook on the diagnostic performance of the staff assessed in their studies was positive, negative or mixed. The results and discussion sections of each study were searched by VS for language that indicated whether authors viewed the diagnostic performance of the staff in their studies in a positive or negative way or whether they had a mixed reaction, and were coded accordingly. The coding was reviewed by PR and revisions were made until consensus was reached.

\section{Results}

The database search yielded 732 results, 353 of which were excluded as duplicates. The titles and abstracts of the remaining 379 were screened and 264 of them were excluded. Full text screening was performed for 115 studies. From those, 47 studies were excluded based on the inclusion/exclusion criteria leaving 68 studies to be included in the review (Figure 1). 


\section{Study characteristics}

The included studies were published between 1989 and 2017, with the number of studies increasing steadily with each passing decade. The majority of the studies took place in Europe, most prominently the UK, Australasia and Eastern Asia. Forty-one of the studies originated from developed countries and 27 from developing economic regions (based on UN country classification available at www.un.org/development/desa/dpad/wpcontent/uploads/sites/45/publication/2015wesp_full_en.pdf) (Table 1).

Most studies ( $n=55$ ) employed a cross-sectional study design, however eleven ${ }^{25,29-31,34,35,38-}$ $40,46,70$ studies were educational interventions with a before and after design and two ${ }^{37,75}$ were RCTs. Sample size varied widely between studies (10-2700 staff tested); Only thirteen ${ }^{27,46,53,56,59,60,64,67,68,71,72,86,87}$ studies reported on how the sample size was calculated. (Appendix)

In the majority of the studies ( $n=43)$, any member of the pharmacy team were the subject of investigation. In some cases, ${ }^{21,25,26,33,41,42,50,52,57,72,78}$ it was not possible to verify staff role, either because the researchers stated they were not able to do so, or because the study did not make it clear. The terminology used to describe 'other' pharmacy staff was divergent and often location-specific and it was not always clear to gauge what role they had or the level of training/qualification they held. All studies except one ${ }^{55}$ (purely qualitative) used quantitative methods to report their results, although four ${ }^{36,48,79,87}$ studies did use a combination of quantitative and qualitative methods. (Table 2)

\section{Quality characteristics}

Risk of bias assessment analysis for the cross-sectional studies rated ten $21,26,33,41,42,50,52,57,72,78$ to be of poor quality and the other 45 to be of fair quality. As these studies had many things in common, in terms of research questions, methodology and outcome measures, the main differentiating factor between fair and poor quality was whether they accurately defined their study population and between fair and good the fact that no studies assessed their population more than once, all studies' outcome measures were not reliable enough and only one study ${ }^{86}$ mentioned that their SPs were blinded to the correct scenario responses.

Assessment for the educational interventions and RCTs found nine studies $25,29,30,31,34,35,37,70,75$ to be of poor quality and four $38,39,40,46$ to be of fair quality with the main reasons for lowering 
quality being non-adequate randomisation, not describing adherence to training protocols and not reporting the statistical power of their main outcomes as well as that, due to their nature, sufficient blinding and training allocation were not possible.

Study piloting was reported to have happened in only $29 \%(n=20)$ of studies. Only one ${ }^{34}$ of the educational interventions reported piloting. Despite low levels of reported piloting, most (71\%, $n=44$ out of 62 ) SP studies, did report on the training provided to SPs prior to data collection. In the vast majority of the studies $(84 \%, n=57)$, data collection forms were completed after pharmacy staff interaction. In 18 studies (26\%) the use of audio or video recording was employed. Twelve (18\%) used both of these methods to be able to verify the content of the collection forms.(Appendix)

\section{Methodology characteristics}

The most prominent methodology used to assess pharmacist and staff performance was through simulated patients; only 5 studies used vignettes, ${ }^{44,47,48,55,62}$ whilst one ${ }^{80}$ study used both. The number of SPs used in the studies ranged from one to more than 10 , however most studies employed one or two SPs $(n=24)$. Similarly, the numbers of scenarios used in the studies ranged from one to 64 , however most used one or two scenarios ( $n=48$ ). A mixture of symptom presentation and product request scenarios $(n=38)$ were most commonly employed, although 20 studies solely used symptom presentation scenarios and 6 studies were product request scenarios only. In the majority of the scenarios $(n=39)$ the SPs presented as themselves and in 19 studies the SP requested advice or product for a third person such as a child, relative or a friend. Nine studies used both types of presentation. (Appendix)

\section{Assessment characteristics}

Many of the studies ( $n=29)$ used published guidelines, recommendations and standards, and a further 10 based their assessment on criteria used in other published studies. Eighteen studies stated that the authors had derived their own criteria, whilst 10 studies used 'expert panels'. Eight studies explicitly mentioned basing their criteria on mnemonic acronyms, most commonly mentioned of which was WWHAM (Who is the patient, What are the symptoms, How long have the symptoms been present, Action taken, Medication being taken). Sixteen studies used some form of scoring system for their results (Table 2). 
The majority of studies $(n=46)$ evaluated staff's performance on specific health conditions whilst the remainder $(n=22)$ assessed general diagnostic performance. Sub-analysis of data comparing condition with country showed that studies conducted in developed economies tended to concentrate on women's health, such as emergency contraception, and central nervous system conditions such as insomnia and headache. Those studies emanating from developing economies concentrated on conditions such as diarrhoea and sexually transmitted diseases (Appendix).

\section{Assessment framework ratings}

Based on our scoring system, studies tended to have, in total, higher mnemonic characteristics in their assessment methods of pharmacy staff's performance, with a modal value of 3 and lower clinical reasoning characteristics with a modal value of 0 . Inter-rater agreement for the clinical reasoning rating was $80.5 \%$ and $82.4 \%$ for the mnemonic rating, before disagreements were resolved through discussion. There were no great differences in modal values between developed and developing economies ratings (clinical reasoning modal value of 0 for both, 2 for developing countries' mnemonic rating and 3 for developed countries' mnemonic rating) or between studies that set out to assess general diagnostic performance or a specific condition (clinical reasoning modal value of 0 in both cases, mnemonic rating modal value of 3 for general performance and 4 for specific conditions) . For each mnemonic framework characteristic individually, 69\% of studies assessed performance based on questions not always relevant to scenario condition, $85 \%$ used checklists, $43 \%$ used named mnemonics or guidelines and $74 \%$ have reported the final decision staff took. For each clinical reasoning framework individually, 53\% of studies assessed performance based on questions with relevance to the condition at hand, $12 \%$ reported purposes for the questions asked, $7 \%$ reflected on how the gathered information was used and $24 \%$ considered a connection between the information gathering process and the decision-making outcome.

\section{Study authors' outlook on performance}

Nine of the 68 studies' authors described pharmacy staff's performance in positive terms (example quote: "Results across all scenarios indicated the provision of a training program [...]

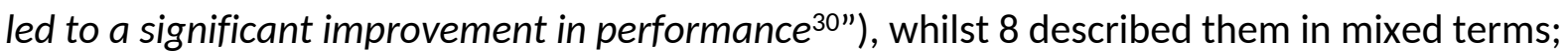


the vast majority of authors $(n=51)$ used negative terms (example quote: "assessment and counselling provided to such patients were inadequate" ${ }^{63}$ ) to describe their results of pharmacy staff's performance. Three of the thirteen educational interventions and RCT studies used positive language compared to 6 of the 55 cross-sectional studies. Three of the four educational interventions that were assessed to be of fair quality used negative language for their results. (Table 5)

\section{Comparisons}

Eleven studies included a theoretical assessment of staff's knowledge (in the form of a questionnaire) which was then compared to actual performance through SPs or vignette methodology. Seven $21-23,35,45,71,86$ reported actual performance was worse than the performance measured with the questionnaires, one ${ }^{41}$ study found them to be similar and the other two ${ }^{25,39}$ did not report that information. In the 13 studies that reported comparisons between community pharmacists and other staff, nine $20,37,43,59,66,67,73,76,83$ reported pharmacists performing better and four $21,60,85,86$ reported similar performances.

\section{Discussion}

Performance of pharmacy staff was overwhelmingly reported as poor by study authors, a result which has been reflected in other reviews ${ }^{88}$. This appeared to be the case regardless of geography, scenario used or whichever assessment framework was utilised. Scrutiny on how authors arrived at these conclusions revealed that they relied on mnemonic criteria to assess pharmacy staff's diagnostic performance rather than a clinical reasoning approach. This means that potentially important aspects of the decision-making process, that clinical reasoning accounts for, were left unexplored.

The mnemonic framework provided simple quantifiable results, such as numbers of questions asked and the ability then to produce a score, however mnemonic characteristics have been called into question in establishing pharmacy staff ability to derive a diagnosis ${ }^{12,13,48}$. The various existing guidelines and well used mnemonics, such as WWHAM (commonly taught in UK pharmacy schools), appear to be viewed as appropriate instruments by authors. However, to aid a better representation of the actual level of diagnostic staff performance, new tools need to be developed, more aligned with a clinical reasoning approach, which would allow for the assessment of all parts of the decision-making process. New tools should expand 
further than measuring the amount of questions being asked based on standardised mnemonic methods and examine whether questions being asked by pharmacists and staff are based on evidence-based knowledge of the scenarios presented to them, what pharmacists or staff want to achieve through their questions and observations and how everything relates to their decision-making process and their final decisions. Because these concepts are very difficult to be described and examined in quantitative terms, more qualitative methods could be employed by researchers, as they could be used in researching community pharmacists' and other staff's thought process during consultations, identify pharmacists', staff's and pharmacy students' needs and attitudes towards improving their diagnostic skills, examine the impact of educational interventions on decision-making and diagnostic abilities and other potential research topics. Qualitative methodology has been underutilised so far, with only four of all included studies in this review using it.

Tools that would be validated, and subsequently more reliable, would help improve the overall quality of future studies and avoid risk of bias. Future studies should also take care in defining their study populations and having their participants and assessors be blinded to the correct scenario responses. Educational interventions aimed at improving diagnosing ability in particular, could employ adequate randomisation, include in their results to what extent protocols are adhered to, report the statistical power of their results and have a longer length and more follow-ups to make sure any positive results can last over time.

Comparisons of pharmacy staff versus pharmacists showed that, on the whole, pharmacists performed better. This is to be expected, as pharmacists have more extensive training than other pharmacy staff, and this finding favours pharmacists being more visible and proactive in undertaking patient consultations rather than leaving this role to less well trained staff ${ }^{9}$. In studies where actual performance was compared to theoretical performance, staff performed more poorly in the real-life situation scenarios. This dissonance suggests that decision making skills of staff are poorer than they perceive, whereby they possess knowledge but do not know how to use it critically. This performance gap is somewhat substantiated through the findings of the educational intervention studies, which showed broadly positive results (Table 5), although these studies were mostly short-term and of not good quality so we cannot draw any firm conclusion about how long these effects may last. However, these comparisons were based on very limited numbers of studies and larger reviews would be needed to substantiate these findings. 


\section{Conclusion}

The current literature assessing pharmacists' and pharmacy staff's diagnostic ability via simulated patients or vignettes overwhelmingly relies on a mnemonic rather than a clinical reasoning framework. Based on authors' comments about their results, the common perception is that pharmacists' and staff's diagnostic ability is poor, regardless of geography, diagnostic scenarios or assessment framework. A limited amount of studies found pharmacists perform better than other pharmacy staff. The quality of future studies can be improved and new tools should be developed for future assessments that go further than measuring the amount of questions asked and deeper examine the decision-making process during consultations. 


\section{References}

1. Department of Health. Research evidence on the effectiveness of self care support. 2007.

2. The task group of the Pharmacy and Public Health Forum. Health on the high street. 2013.

3. The World Self-Medication Industry. Responsible Self-Care and Self-Medication. A Worldwide Review of Consumer Surveys. Available at: http://www.wsmi.org/wpcontent/data/pdf/wsmibro3.pdf, 2016.

4. Royal Pharmaceutical Society | Improving urgent and emergency care through better use of pharmacists. Available at: http://www.rpharms.com/our-campaigns/improving-urgentand-emergency-care-through-better-use-of-pharmacists.asp. Accessed 10/5/2016, 2016.

5. Nichol, M. B., McCombs, J. S., Johnson, K. A., Spacapan, S., \& Sclar, D. A. (1992). The effects of consultation on over-the-counter medication purchasing decisions. Medical care, 9891003.6. A Sclar BPharm, D., M Robison, L., \& L Skaer BPharm, T. (1996). Pharmacy consultation and over-the-counter medication purchasing outcomes. Journal of clinical pharmacy and therapeutics, 21(3), 177-184.7. Hassell, K., Whittington, Z., Cantrill, J., Bates, F., Rogers, A., \& Noyce, P. (2001). Managing demand: transfer of management of self limiting conditions from general practice to community pharmacies. Bmj, 323(7305), 146-147.8. Rosenthal, M., Austin, Z., \& Tsuyuki, R. T. (2010). Are pharmacists the ultimate barrier to pharmacy practice change?. Canadian Pharmacists Journal/Revue des Pharmaciens du Canada, 143(1), 37-42.

9. Ward, P. R., Bissell, P., \& Noyce, P. R. (1998). Medicines counter assistants: roles and responsibilities in the sale of deregulated medicines. International Journal of Pharmacy Practice, 6(4), 207-215.

10. Emmerton, L., \& Shaw, J. (2002). The influence of pharmacy staff in non-prescription medicine sales. International Journal of Pharmacy Practice, 10(2), 101-106.

11. Shealy, K. M. (2014). Mnemonics to assess patients for self-care: is there a need?. SelfCare Journal, 5(1), 11-18.

12. Rutter, P., \& Patel, J. (2013). Decision making by community pharmacists when making an over-the-counter diagnosis in response to a dermatological presentation. Self Care, 4(6), 125133.

13. Iqbal, N., \& Rutter, P. (2013). Community pharmacists reasoning when making a diagnosis: a think-aloud study. International Journal of Pharmacy Practice, 21, 17-17. 
14. Sinopoulou, V., Summerfield, P., \& Rutter, P. (2017). A qualitative study on community pharmacists' decision-making process when making a diagnosis. Journal of evaluation in clinical practice, 23(6), 1482-1488.

15. Norman, G. (2005). Research in clinical reasoning: past history and current trends. Medical education, 39(4), 418-427.

16. Ramezani-Badr, F., Nasrabadi, A. N., Yekta, Z. P., \& Taleghani, F. (2009). Strategies and criteria for clinical decision making in critical care nurses: a qualitative study. Journal of Nursing Scholarship, 41(4), 351-358.

17. Han, K. J., Kim, H. S., Kim, M. J., Hong, K. J., Park, S., Yun, S. N., ... \& Choi, H. (2007). Thinking in clinical nursing practice: a study of critical care nurses' thinking applying the think-aloud, protocol analysis method. Asian nursing research, 1(1), 68-82.

18. May, S., Greasley, A., Reeve, S., \& Withers, S. (2008). Expert therapists use specific clinical reasoning processes in the assessment and management of patients with shoulder pain: a qualitative study. Australian Journal of Physiotherapy, 54(4), 261-266.

19. Brata, C., Gudka, S., Schneider, C. R., Everett, A., Fisher, C., \& Clifford, R. M. (2013). A review of the information-gathering process for the provision of medicines for selfmedication via community pharmacies in developing countries. Research in social and administrative pharmacy, 9(4), 370-383.

20. Scully, C., Gill, Y., \& Gill, Z. (1989). How community pharmacy staff manage a patient with possible oral cancer. British Journal of Oral and Maxillofacial Surgery, 27(1), 16-21.

21. Aktekin, M., Erozgen, C., \& Donmez, L. (1998). Pharmacy approach to a case of acute diarrhoea with dehydration in Antalya, Turkey. Public health, 112(5), 323-326.

22. Anderson, C., Alexander, A. (1993). Response to dysmenorrhoea: an assessment of pharmacists' knowledge and its application in practice. International Journal of Pharmacy Practice, 2(3), 180-183.

23. Goodburn, E., Mattosinho, S., Mongi, P., \& Waterston, T. (1991). Management of childhood diarrhoea by pharmacists and parents: is Britain lagging behind the Third World?. BMJ, 302(6774), 440-443.

24. Lamsam, G. D., \& Kropff, M. A. (1998). Community pharmacists' assessments and recommendations for treatment in four case scenarios. Annals of Pharmacotherapy, 32(4), 409-416. 
25. Ross-Degnan, D., Soumerai, S. B., Goel, P. K., Bates, J., Makhulo, J., Dondi, N. et al (1996). The impact of face-to-face educational outreach on diarrhoea treatment in pharmacies. Health policy and planning, 11(3), 308-318.

26. Wachter, D. A., Joshi, M. P., \& Rimal, B. (1999). Antibiotic dispensing by drug retailers in Kathmandu, Nepal. Tropical Medicine \& International Health, 4(11), 782-788.

27. Alte, D., Weitschies, W., \& Ritter, C. A. (2007). Evaluation of consultation in community pharmacies with mystery shoppers. Annals of Pharmacotherapy, 41(6), 1023-1030.

28. Anderson, C., \& Bissell, P. (2004). Using semi covert research to evaluate an emergency hormonal contraception service. Pharmacy World and Science, 26(2), 102-106.

29. Benrimoj, S. I., Gilbert, A. L., de Almeida Neto, A. C., \& Kelly, F. (2009). National implementation of standards of practice for non-prescription medicines in Australia. Pharmacy world \& science, 31(2), 230-237.

30. Benrimoj, S. C. I., Gilbert, A., Quintrell, N., \& de Almeida Neto, A. C. (2007). Nonprescription medicines: a process for standards development and testing in community pharmacy. Pharmacy world \& science, 29(4), 386-394.

31. Berger, K., Eickhoff, C., \& Schulz, M. (2005). Counselling quality in community pharmacies: implementation of the pseudo customer methodology in Germany. Journal of clinical pharmacy and therapeutics, 30(1), 45-57.

32. Kwena, Z. A., Sharma, A., Muga, C., Wamae, N., \& Bukusi, E. A. (2008). Management of simulated patients with sexually transmitted infections by staff of retail pharmacies in Kibera slums of Nairobi. East African medical journal, 85(9), 419-424.

33. Norris, P. T. (2002). Purchasing restricted medicines in New Zealand pharmacies: results from a" mystery shopper" study. Pharmacy World \& Science, 24(4), 149-153.

34. Puumalainen, I. I., Peura, S. H., Kansanaho, H. M., Benrimoj, C. S., \& Airaksinen, M. S. (2005). Progress in patient counselling practices in Finnish community pharmacies. International Journal of Pharmacy Practice, 13(2), 149-156.

35. Rogers, S. N., Lowe, D., Catleugh, M., \& Edwards, D. (2010). An oral cancer awareness intervention in community pharmacy. British Journal of Oral and Maxillofacial Surgery, 48(7), 498-502.

36. Rutter, P. M., Horsley, E., \& Brown, D. T. (2004). Evaluation of community pharmacists' recommendations to standardized patient scenarios. 
37. Watson, M. C., Bond, C. M., Grimshaw, J., \& Johnston, M. (2006). Factors predicting the guideline compliant supply (or non-supply) of non-prescription medicines in the community pharmacy setting. BMJ Quality \& Safety, 15(1), 53-57.

38. Watson, M. C., Cleland, J. A., \& Bond, C. M. (2009). Simulated patient visits with immediate feedback to improve the supply of over-the-counter medicines: a feasibility study. Family practice, 26(6), 532-542.

39. Watson, M. C., Cleland, J., Inch, J., Bond, C. M., \& Francis, J. (2007). Theory-based communication skills training for medicine counter assistants to improve consultations for non-prescription medicines. Medical education, 41(5), 450-459.

40. Adu-Sarkodie, Y., Steiner, M. J., Attafuah, J., \& Tweedy, K. (2000). Syndromic management of urethral discharge in Ghanaian pharmacies. Sexually transmitted infections, 76(6), 439-442. 41. Chalker, J., Chuc, N. T. K., Falkenberg, T., Do, N. T., \& Tomson, G. (2000). STD management by private pharmacies in Hanoi: practice and knowledge of drug sellers. Sexually transmitted infections, 76(4), 299-302.

42. Driesen, A., \& Vandenplas, Y. (2009). How do pharmacists manage acute diarrhoea in an 8 month-old baby? A simulated client study. International Journal of Pharmacy Practice, 17(4), 215-220.

43. Evans, M. J., \& Gibbons, A. J. (2005). Advice given in community pharmacies to patients with possible oral carcinoma. British Journal of Oral and Maxillofacial Surgery, 43(3), 253-255. 44. Karim, R., Ramdahin, P., Boodoo, J. R., Kochhar, A., \& Pinto Pereira, L. M. (2004). Community pharmacists' knowledge and dispensing recommendations for treatment of acute diarrhoea in Trinidad, West Indies. International journal of clinical practice, 58(3), 264-267. 45. Leiva, A., Shaw, M., Paine, K., Manneh, K., McAdam, K., \& Mayaud, P. (2001). Management of sexually transmitted diseases in urban pharmacies in The Gambia. International journal of STD \& AIDS, 12(7), 444-452.

46. Sigrist, T., Benrimoj, S. I., Hersberger, K., \& Langford, J. (2002). Changing pharmacists' and pharmacist assistants' practice in dealing with direct requests for non-prescription analgesics. International Journal of Pharmacy Practice, 10(1), 23-29. 
47. Turner, A. N., Ellertson, C., Thomas, S., \& García, S. (2003). Diagnosis and treatment of presumed STIs at Mexican pharmacies: survey results from a random sample of Mexico City pharmacy attendants. Sexually transmitted infections, 79(3), 224-228.

48. Akhtar, S., \& Rutter, P. (2015). Pharmacists thought processes in making a differential diagnosis using a gastro-intestinal case vignette. Research in Social and Administrative Pharmacy, 11(3), 472-479.

49. Alabid, A. H. M., Ibrahim, M. I. M., \& Hassali, M. A. (2013). Do professional practices among malaysian private healthcare providers differ? A comparative study using simulated patients. Journal of clinical and diagnostic research: JCDR, 7(12), 2912.

50. Bardage, C., Westerlund, T., Barzi, S., \& Bernsten, C. (2013). Non-prescription medicines for pain and fever-a comparison of recommendations and counseling from staff in pharmacy and general sales stores. Health Policy, 110(1), 76-83.

51. Bilkhu, P. S., Wolffsohn, J. S., Tang, G. W., \& Naroo, S. A. (2014). Management of dry eye in UK pharmacies. Contact Lens and Anterior Eye, 37(5), 382-387.

52. Bilkhu, P., Wolffsohn, J. S., Taylor, D., Gibson, E., Hirani, B., \& Naroo, S. A. (2013). The management of ocular allergy in community pharmacies in the United Kingdom. International journal of clinical pharmacy, 35(2), 190-194.

53. Chalker, J. C., Vialle-Valentin, C., Liana, J., Mbwasi, R., Semali, I. A., Kihiyo, B., et al (2015). What roles do accredited drug dispensing outlets in Tanzania play in facilitating access to antimicrobials? Results of a multi-method analysis. Antimicrobial resistance and infection control, 4(1), 33.

54. Chua, S. S., Ramachandran, C. D., \& Paraidathathu, T. T. (2006). Response of community pharmacists to the presentation of back pain: a simulated patient study. International Journal of Pharmacy Practice, 14(3), 171-178.

55. Chui, M. A., Stone, J. A., Martin, B. A., Croes, K. D., \& Thorpe, J. M. (2013). Safeguarding older adults from inappropriate over-the-counter medications: the role of community pharmacists. The Gerontologist, 54(6), 989-1000.

56. da Rocha, C. E., Bispo, M. L., dos Santos, A. C. O., Mesquita, A. R., Brito, G. C., \& de Lyra Jr, D. P. (2015). Assessment of community pharmacists' counseling practices with simulated patients who have minor illness: A Pilot Study. Simulation in healthcare, 10(4), 227-238. 
57. Higgins, S. J., \& Hattingh, H. L. (2013). Requests for emergency contraception in community pharmacy: An evaluation of services provided to mystery patients. Research in Social and Administrative Pharmacy, 9(1), 114-119.

58. Horvat, N., Koder, M., \& Kos, M. (2012). Using the simulated patient methodology to assess paracetamol-related counselling for headache. PLoS One, 7(12), e52510.

59. Hussain, A., Ibrahim, M. I., \& Malik, M. (2013). Assessment of disease management of insomnia at community pharmacies through simulated visits in Pakistan. Pharmacy practice, 11(4), 179

60. Hussain, A., Ibrahim, M. I., \& Malik, M. (2012). Assessment of disease management of acute respiratory tract infection at community pharmacies through simulated visits in Pakistan. Latin Am J Pharm, 31(10), 1435-40.

61. Hussain, A., Shafiq, M., Khuwaja, W., Qayyum, M. I., Irshad, N., \& Nadeem, M. (2013). Management of Peptic Ulcer Disease at Community Pharmacies in Pakistan by Using Simulated Patient Visits. International Journal of Pharmaceutical Sciences Review and Research, 21(1), 15-9.

62. Jiwa, M., Spilsbury, K., \& Duke, J. (2010). Do pharmacists know which patients with bowel symptoms should seek further medical advice? A survey of pharmacists practicing in community pharmacy in Western Australia. Annals of Pharmacotherapy, 44(5), 910-917.

63. Kashour, T. S., Joury, A., Alotaibi, A. M., Althagafi, M., Almufleh, A. S., Hersi, A., \& Thalib, L. (2016). Quality of assessment and counselling offered by community pharmacists and medication sale without prescription to patients presenting with acute cardiac symptoms: a simulated client study. European journal of clinical pharmacology, 72(3), 321-328.

64. Kippist, C., Wong, K., Bartlett, D., \& Saini, B. (2011). How do pharmacists respond to complaints of acute insomnia? A simulated patient study. International journal of clinical pharmacy, 33(2), 237-245.

65. MacFarlane, B., Bergin, J., \& Peterson, G. M. (2016). Assessment and management of serotonin syndrome in a simulated patient study of Australian community pharmacies. Pharmacy Practice (Granada), 14(2), 0-0.

66. MacFarlane, B., Matthews, A., \& Bergin, J. (2015). Non-prescription treatment of NSAID induced GORD by Australian pharmacies: a national simulated patient study. International journal of clinical pharmacy, 37(5), 851-856. 
67. Malik, M., Hassali, M. A., Shafie, A. A., Hussain, A., Aljadhey, H., \& Saleem, F. (2013). Case management of malaria fever at community pharmacies in Pakistan: a threat to rational drug use. Pharmacy practice, 11(1), 8.

68. Malik, M., Hussain, A., Shafiq, M., \& Iqbal, J. (2013). Assessment of case management of dyspepsia at community pharmacies in two cities of Pakistan. Marmara Pharmaceutical Journal, 17(1), 7-11.

69. Mesquita, A. R., de Oliveira Sá, D. A. B., Santos, A. P. A. L., de Almeida Neto, A., \& Lyra, D. P. (2013). Assessment of pharmacist's recommendation of non-prescription medicines in Brazil: a simulated patient study. International journal of clinical pharmacy, 35(4), 647-655.

70. Murphy, A. L., Martin-Misener, R., Kutcher, S. P., \& Gardner, D. M. (2015). Pharmacists' performance in a telephone-based simulated patient study after a mental health capacitybuilding program. International journal of clinical pharmacy, 37(6), 1009-1013.

71. Ogbo, P. U., Aina, B. A., \& Aderemi-Williams, R. I. (2014). Management of acute diarrhea in children by community pharmacists in Lagos, Nigeria. Pharmacy practice, 12(1).

72. Puspitasari, H. P., Faturrohmah, A., \& Hermansyah, A. (2011). Do Indonesian community pharmacy workers respond to antibiotics requests appropriately?. Tropical Medicine \& International Health, 16(7), 840-846.

73. Sancar, M., Tezcan, E., Okuyan, B., \& Izzettin, F. V. (2015). Assessment of the attitude of community pharmacists and pharmacy technicians towards diarrhea: a simulated patient study in Turkey. Tropical Journal of Pharmaceutical Research, 14(8), 1509-1515.

74. Santos, A. P., Mesquita, A. R., Oliveira, K. S., \& Lyra Jr, D. P. (2013). Assessment of community pharmacists' counselling skills on headache management by using the simulated patient approach: a pilot study. Pharmacy practice, 11(1), 3.

75. Schneider, C. R., Emery, L., Brostek, R., \& Clifford, R. M. (2013). Evaluation of the supply of antifungal medication for the treatment of vaginal thrush in the community pharmacy setting: a randomized controlled trial. Pharmacy practice, 11(3), 132.

76. Schneider, C. R., Everett, A. W., Geelhoed, E., Kendall, P. A., Murray, K., Garnett, P., ... \& Clifford, R. M. (2011). Provision of primary care to patients with chronic cough in the community pharmacy setting. Annals of Pharmacotherapy, 45(3), 402-408.

77. Schneider, C. R., Gudka, S., Fleischer, L., \& Clifford, R. M. (2013). The use of a written assessment checklist for the provision of emergency contraception via community pharmacies: a simulated patient study. Pharmacy practice, 11(3), 127. 
78. van Hoof, J. J., Cents, M. H., Megens, N. M., \& van der Tang, S. J. (2014). Druggists and pharmacists as gatekeepers: Sales routines and compliance with sales protocols for over-thecounter naproxen 275 mg medicines in the Netherlands. Health policy, 117(3), 353-360.

79. Veiga, P., Lapão, L. V., Cavaco, A. M., \& Guerreiro, M. P. (2015). Quality supply of nonprescription medicines in Portuguese community pharmacy: an exploratory case study. Research in Social and Administrative Pharmacy, 11(6), 880-890.

80. Vu, D. H., van Rein, N., Cobelens, F. G. J., Nguyen, T. T. H., Le, V. H., \& Brouwers, J. R. B. J. (2012). Suspected tuberculosis case detection and referral in private pharmacies in Viet Nam. The International Journal of Tuberculosis and Lung Disease, 16(12), 1625-1629.

81. Xu, T., de Almeida Neto, A. C., \& Moles, R. J. (2012). Simulated caregivers: their feasibility in educating pharmacy staff to manage children's ailments. International journal of clinical pharmacy, 34(4), 587-595.

82. Byrne, G. A., Wood, P. J., \& Spark, M. J. (2017). Non-prescription supply of combination analgesics containing codeine in community pharmacy: A simulated patient study. Research in Social and Administrative Pharmacy.

83. Hussain, A., \& Ibrahim, M. I. M. (2012). Management of diarrhoea cases by community pharmacies in 3 cities of Pakistan/Prise en charge des cas de diarrhée en pharmacies communautaires dans trois villes du Pakistan. Eastern Mediterranean Health Journal, 18(6), 635.

84. Inch, J., Porteous, T., Maskrey, V., Blyth, A., Burr, J., Cleland, J., ... \& Watson, M. C. (2017). It's not what you do it's the way that it's measured: quality assessment of minor ailment management in community pharmacies. International Journal of Pharmacy Practice, 25(4), 253-262.

85. Kashyap, K. C., Nissen, L. M., Smith, S. S., \& Kyle, G. (2014). Management of over-thecounter insomnia complaints in Australian community pharmacies: a standardized patient study. International Journal of Pharmacy Practice, 22(2), 125-134.

86. Saengcharoen, W., \& Lerkiatbundit, S. (2010). Practice and attitudes regarding the management of childhood diarrhoea among pharmacies in Thailand. International Journal of Pharmacy Practice, 18(6), 323-331.

87. Queddeng, K., Chaar, B., \& Williams, K. (2011). Emergency contraception in Australian community pharmacies: A simulated patient study. Contraception, 83(2), 176-182. 
88. van Eikenhorst, L., Salema, N. E., \& Anderson, C. (2017). A systematic review in select countries of the role of the pharmacist in consultations and sales of non-prescription medicines in community pharmacy. Research in Social and Administrative Pharmacy, 13(1), 17-38. 
Table 1 Publication year and country

\begin{tabular}{|l|l|l|}
\hline Criterion & Studies & References \\
\hline Year & & \\
\hline 1989 & 1 & 20 \\
\hline $1990-1999$ & 6 & $21,22,23,24,25,26$ \\
\hline $2000-2009$ & 21 & $27,28,29,30,31,32,33,34,35,36,37,38,39$, \\
& & $40,41,42,43,44,45,46,47$ \\
\hline $2010-2017$ & 40 & $48,49,50,51,52,53,54,55,56,57,58,59,60,61,62,63,64$, \\
& & $65,66,67,68,69,70,71,72,73,74,75,76,77,78,79,80,81$ \\
\hline Country & & $82,83,84,85,86,87$ \\
\hline & & \\
\hline UK & 14 & $13-15,20,22,28,35,36,43,48,51,52,84$ \\
\hline Europe & 9 & $27,31,34,42,46,50,58,78,79$ \\
\hline Eastern Asia & 13 & $26,41,49,54,59-61,67,68,72,80,83,86$ \\
\hline Western Asia & 3 & $21,73,63$ \\
\hline Northern America & 4 & $24,47,55,70$ \\
\hline Southern America & 4 & $44,56,69,74$ \\
\hline Australasia & 15 & $29,30,33,57,62,64,65,66,75-77,81,82,85,87$ \\
\hline Africa & 5 & $32,40,45,53,71$ \\
\hline Transnational & 1 & 25 \\
\hline developing countries & 27 & $21,25,26,32,40,41,44,45,47,49,53,54,56,59-$ \\
\hline developed countries & & $61,63,67,68,69,71,72-74,80,83,86$ \\
\hline & 41 & $20,22-24,27-31,33-39,42,43,46,48,50-52,55,60,62,64-$ \\
\hline
\end{tabular}

Table 2 Other study characteristics

\begin{tabular}{|l|l|l|}
\hline $\begin{array}{l}\text { Sample } \\
\text { characteristics }\end{array}$ & studies & references \\
\hline CPs only & 18 & $22-24,28,36,44,48,54-56,58,62-64,69-71,74$ \\
\hline Any pharmacy staff & 44 & $\begin{array}{l}20,21,26,27,29-31,33-35,37,38,40-43,46,47,50-52,57,59-61,65-68, \\
72,73,75-87\end{array}$ \\
\hline Non-CP staff only & 5 & $25,32,39,45,53$ \\
\hline CPs and GPs & 1 & 49 \\
\hline Type of results & & all studies except 55 \\
\hline $\begin{array}{l}\text { Quantitatively } \\
\text { (descriptive or } \\
\text { more advanced } \\
\text { statistics) }\end{array}$ & 67 & $24,27,30,34,38,46,58-60,64,68,69,79,81-83$ \\
\hline $\begin{array}{l}\text { Quantitatively } \\
\text { (using a scoring } \\
\text { system) }\end{array}$ & 16 & 55 \\
\hline $\begin{array}{l}\text { Qualitatively } \\
\text { (themes) }\end{array}$ & 1 & $36,48,79,87$ \\
\hline $\begin{array}{l}\text { Both quantitatively } \\
\text { and qualitatively }\end{array}$ & 4 & \begin{tabular}{l} 
(CP=Community Pharmacist, GP=General Practitioner) \\
\hline
\end{tabular} \\
\hline
\end{tabular}

(CP=Community Pharmacist, GP=General Practitioner) 
Table 3 Assessment criteria basis

\begin{tabular}{|l|l|l|}
\hline $\begin{array}{l}\text { Assessment criteria were decided based } \\
\text { on: }\end{array}$ & & \\
\hline author derived & 18 & $22,23,31,34,35,43-48,57,72,75,79,82,85$ \\
\hline paper & 10 & $49,50,52,62,64-66,69,70,74$ \\
\hline standards/guidelines/recommendations & 29 & $\begin{array}{l}25,28-30,32,34,37,39,44,47,51,54,57-61,64,68, \\
71,75-78,83,84,86,87\end{array}$ \\
\hline WWHAM protocol & 6 & $37,38,39,42,54,84$ \\
\hline WHAT-STOP-GO protocol & 1 & 81 \\
\hline other protocols & 1 & 54 \\
\hline expert panel & 10 & $24,33,36,38,56,62,64,69,84,86$ \\
\hline n/a & 9 & $20,21,27,41,45,53,63,73,80$ \\
\hline
\end{tabular}

Table 4 Mode score values per characteristic and median values for total scores

\begin{tabular}{|l|l|l|l|l|l|l|l|l|l|l|}
\hline characteristic & CR1 & CR2 & CR3 & CR4 & MN1 & MN2 & MN3 & MN4 & $\begin{array}{l}\text { Total } \\
\text { CR }\end{array}$ & $\begin{array}{l}\text { Total } \\
\text { MN }\end{array}$ \\
\hline mode & 1 & 0 & 0 & 0 & 1 & 1 & 0 & 1 & & \\
\hline median & & & & & & & & & 1 & 3 \\
\hline
\end{tabular}

(CR=clinical reasoning, $M N=$ mnemonic)

Table 5 Authors outlook on staff's performance results

\begin{tabular}{|l|l|l|}
\hline Authors' outlook on diagnostic performance & $n$ & $\begin{array}{l}\text { reference }{ }^{*} \text { denotes educational } \\
\text { intervention) }\end{array}$ \\
\hline positive & 9 & $28,29^{*}, 30^{*}, 37^{*}, 46^{*}, 53,55,65,66$ \\
\hline mixed & 8 & $27,31^{*}, 58,62,70^{*}, 79,80,84$ \\
\hline negative & 51 & $\begin{array}{l}20-24,25^{*}, 32,33,34^{*}, 35^{*}, 36,38^{*}, \\
39^{*}, 40^{*}, 41-45,47-52,54,56,57,59- \\
\end{array}$ \\
& & $\begin{array}{l}61,63,64,67-69,71-74,75^{*}, 76-78, \\
81-83,85-87\end{array}$ \\
\hline
\end{tabular}




\section{Search results from MEDLINE, EMBASE, Web of Science and} manual searching

\section{$\mathrm{N}=\mathbf{7 3 2}$ (22 through manual search)}

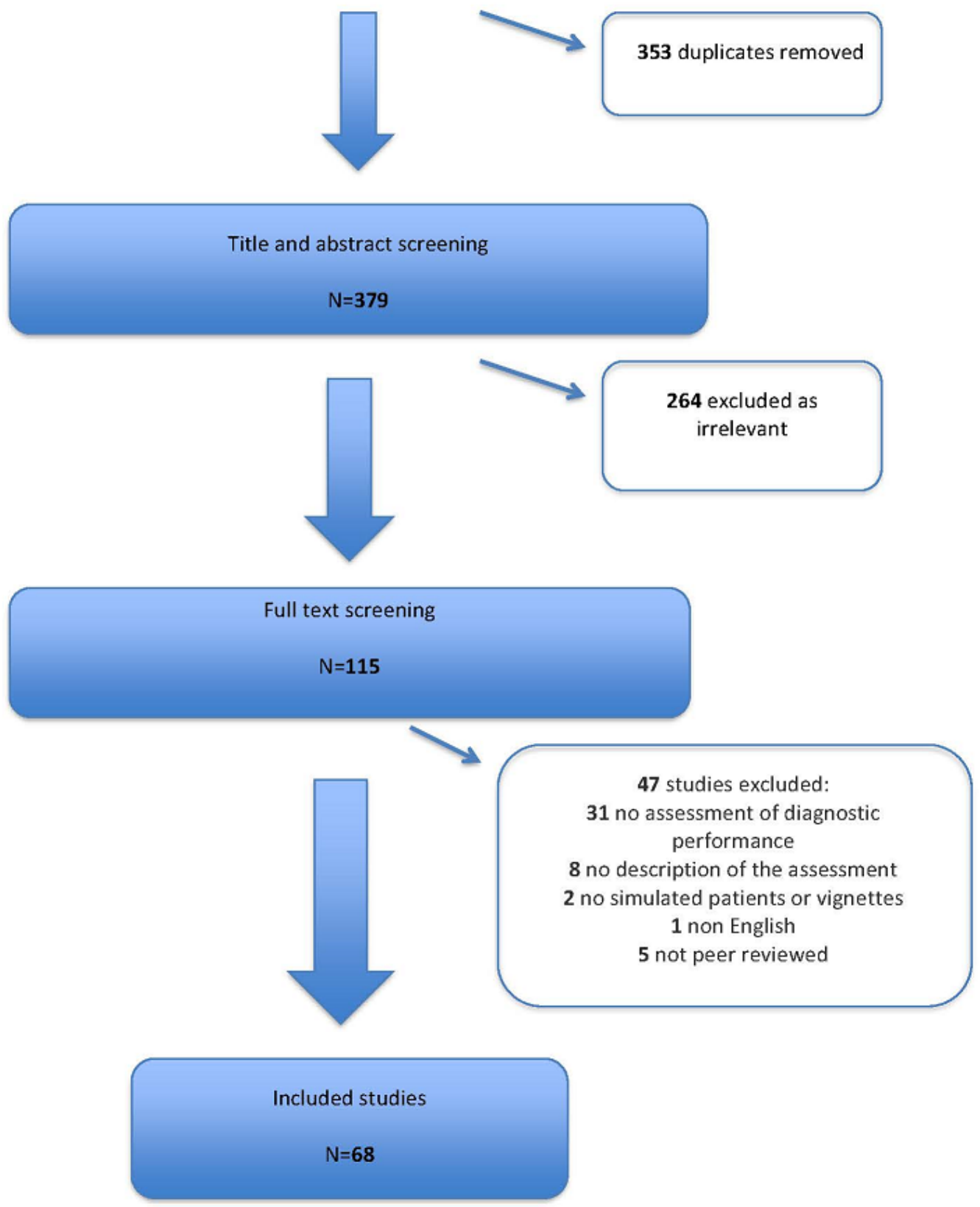

Figure 1: Inclusion and Exclusion Flow Chart 


\section{Appendix}

Table 6 Types of studies and sample sizes

\begin{tabular}{|c|c|c|c|c|c|}
\hline Type of study & Studies & References & $\begin{array}{l}\text { Sample } \\
\text { size }\end{array}$ & Studies & References \\
\hline $\begin{array}{l}\text { Cross- } \\
\text { sectional }\end{array}$ & 55 & $\begin{array}{l}20-24,26-28, \\
32,33,36,41-45 \\
47-49,50-69 \\
71-74,76-87\end{array}$ & $\begin{array}{l}<100: \\
\text { 101-200: } \\
\text { 201-300: } \\
>301:\end{array}$ & $\begin{array}{l}28 \\
12 \\
8 \\
7\end{array}$ & $\begin{array}{l}20,22,23,28,32,36,43-45,48,51,52,54- \\
58,61,64,69,73,74,77,79,81,84,85,87 \\
24,26,42,47,49,62,65,71,76,80,82,86 \\
21,27,41,66-68,72,78 \\
33,50,53,59,60,63,83\end{array}$ \\
\hline $\begin{array}{l}\text { Educational } \\
\text { interventions }\end{array}$ & 11 & $\begin{array}{l}25,29-31,34,35 \\
38-40,46,70\end{array}$ & $\begin{array}{l}<100: \\
\text { 101-200: } \\
\text { 201-300: } \\
>301:\end{array}$ & $\begin{array}{l}5 \\
2 \\
1 \\
3\end{array}$ & $\begin{array}{l}31,38,39,40,70 \\
35,46 \\
34 \\
25,29,30\end{array}$ \\
\hline $\begin{array}{l}\text { Randomised } \\
\text { Controlled } \\
\text { Trials (RCTs) }\end{array}$ & 2 & 37,75 & $\begin{array}{l}<100: \\
>301:\end{array}$ & $\begin{array}{l}1 \\
1\end{array}$ & $\begin{array}{l}75 \\
37 \\
\end{array}$ \\
\hline
\end{tabular}

Table 7 Quality characteristics

\begin{tabular}{|c|c|c|}
\hline Criterion & Studies & References \\
\hline \multicolumn{3}{|l|}{$\begin{array}{l}\text { Was there a pilot to the } \\
\text { study reported by the } \\
\text { authors? }\end{array}$} \\
\hline yes & 21 & $22,26,33,34,36,42,44,48-50,54,56,58,62,63,71,76,77,80,82,87$ \\
\hline no & 47 & $\begin{array}{l}20,21,23-25,27-32,35,37-41,43,45-47,51-53,55,57,59-61, \\
64-70,72-75,78,79,81,83-86\end{array}$ \\
\hline \multicolumn{3}{|l|}{$\begin{array}{l}\text { Did SPs* receive training for } \\
\text { the study? }\end{array}$} \\
\hline yes & 54 & \\
\hline training mentioned & 44 & $24,25,27-30,33-41,45,46,49,52,53,56-60,65,69,70,72-87$ \\
\hline $\begin{array}{r}\text { the SPs had previous } \\
\text { experience }\end{array}$ & 5 & $20,42,66,67,68$ \\
\hline $\begin{array}{r}\text { One or more of the authors } \\
\text { were SPs }\end{array}$ & 6 & $26,22,31,43,54,61$ \\
\hline $\begin{array}{r}\text { external paid service was } \\
\text { used }\end{array}$ & 1 & 50 \\
\hline not mentioned & 6 & $21,23,26,32,51,63$ \\
\hline \multicolumn{3}{|l|}{ How was data captured? } \\
\hline $\begin{array}{l}\text { audio/video recorded and } \\
\text { transcribed }\end{array}$ & 18 & $28,30,33,34,38,39,42,46,48,55,56,58,65,66,69,74,79,84$ \\
\hline data collection form & 57 & $20-27,30-45,47,51,54,57-64,67-87$ \\
\hline paid service & 1 & 50 \\
\hline
\end{tabular}




\begin{tabular}{|l|l|l|}
\hline $\mathrm{n} / \mathrm{a}$ & 3 & $29,52,53$ \\
\hline
\end{tabular}

(SP=Simulated Patient)

Table 8 Methodology characteristics

\begin{tabular}{|c|c|c|}
\hline Methodology & Studies & References \\
\hline $\mathrm{SP}^{*} \mathrm{~S}$ & 62 & $20-43,45,46,49-54,56-61,63-79,81-87$ \\
\hline vignettes & 5 & $44,47,48,55,62$ \\
\hline both & 1 & 80 \\
\hline \multicolumn{3}{|l|}{ Number of SPs } \\
\hline 1 & 13 & $20,22,23,26,31,36,43,45,54,64,70,87$ \\
\hline 2 & 11 & $24,28,32,42,51,56,57,69,73,75$ \\
\hline 3 & 4 & $21,58,77,78$ \\
\hline 4 & 4 & $52,76,82,86$ \\
\hline 5 & 1 & 41 \\
\hline 6 & 4 & $27,65,72,81$ \\
\hline 7 & 2 & 70,72 \\
\hline 8 & 1 & 71 \\
\hline 9 & 1 & 37 \\
\hline 10 & 4 & $40,67,80,84$ \\
\hline$>10$ & 4 & $33,49,50,63$ \\
\hline $\mathrm{n} / \mathrm{a}$ & 12 & $25,29,30,46,53,60,61,66,68,79,83$ \\
\hline \multicolumn{3}{|l|}{ Number of Scenarios } \\
\hline 1 & 32 & $\begin{array}{l}20-23,25,27,35,41-45,48,49,51,52,59,60,64-68,70,71,74, \\
76,77,80,83,86,87\end{array}$ \\
\hline 2 & 16 & $26,28,31,32,33,36,46,55,56,57,63,69,73,75,82,85$ \\
\hline 3 & 7 & $30,38,47,50,53,58,72$ \\
\hline 4 & 5 & $24,34,39,78,84$ \\
\hline$\geq 5$ & 4 & $37,62,79,81$ \\
\hline $\mathrm{n} / \mathrm{a}$ & 4 & $21,29,61,40$ \\
\hline \multicolumn{3}{|l|}{ Type of scenario } \\
\hline Symptom presentation & 20 & $20,21,23,25,26,28,35,36,40,42,43,45,47-49,51,52,65,71,73$ \\
\hline Product request & 6 & $46,64,75,77,82,87$ \\
\hline both & 38 & $\begin{array}{l}22,24,27,30-34,37-39,41,44,50,53-58,60,62,66-70,72,74,78- \\
80,83-86\end{array}$ \\
\hline $\mathrm{n} / \mathrm{a}$ & 4 & $29,59,61,63$ \\
\hline \multicolumn{3}{|l|}{ SP role } \\
\hline for themselves & 32 & $\begin{array}{l}22,27,28,31-33,36-38,40,45,46,49,56,58-61,63,64,66-68 \\
70,74-77,80,82,85,87\end{array}$ \\
\hline $\begin{array}{l}\text { for someone else (child, } \\
\text { relative, friend) }\end{array}$ & 19 & $20,21,23,25,35,41-43,51-54,65,71,78,81,83,84,86$ \\
\hline both & 9 & $24,26,3450,57,69,72,73,79$ \\
\hline $\mathrm{n} / \mathrm{a}$ & 3 & $29,30,39$ \\
\hline
\end{tabular}

(SP=simulated patient) 
Table 9 Conditions for general performance assessment or specific condition

\begin{tabular}{|c|c|c|}
\hline Criterion & Studies & References $\left({ }^{*}\right.$ denotes developing country) \\
\hline \multicolumn{3}{|c|}{$\begin{array}{l}\text { Studies assessing diagnostic ability } \\
\text { of a specific condition }\end{array}$} \\
\hline \multicolumn{3}{|c|}{ women's health } \\
\hline Contraceptive management & 4 & $28,57,77,78$ \\
\hline \multicolumn{3}{|l|}{$\begin{array}{l}\text { Diseases of the genitourinary } \\
\text { system }\end{array}$} \\
\hline dysmenorrhoea & 1 & 22 \\
\hline \multicolumn{3}{|l|}{ Diseases of the visual system } \\
\hline dry eye & 1 & 51 \\
\hline allergic conjuctivitis & 1 & 52 \\
\hline \multicolumn{3}{|l|}{$\begin{array}{l}\text { Diseases of the muscoskeletal } \\
\text { system }\end{array}$} \\
\hline back pain & 1 & $54^{*}$ \\
\hline \multicolumn{3}{|l|}{ Diseases of the nervous system } \\
\hline headache & 2 & $58,74^{*}$ \\
\hline insomnia & 4 & $59^{*}, 64,70,85$ \\
\hline serotonin syndrome & 1 & 65 \\
\hline \multicolumn{3}{|c|}{ Diseases of the respiratory system } \\
\hline Acute respiratory infection & 1 & $60^{*}$ \\
\hline chronic cough & 1 & 76 \\
\hline common cold & 1 & 81 \\
\hline \multicolumn{3}{|l|}{ Diseases of the digestive system } \\
\hline peptic ulcer & 1 & $61^{*}$ \\
\hline lower bowel symptoms & 1 & 62 \\
\hline GORD & 1 & 66 \\
\hline dyspepsia & 1 & $68^{*}$ \\
\hline diarrhoea & 9 & $21^{*}, 23,25^{*}, 42,44^{*}, 71^{*}, 73^{*}, 83^{*}, 86^{*}$ \\
\hline \multicolumn{3}{|c|}{ Conditions related to sexual health } \\
\hline $\begin{array}{l}\text { gonnorhoea and genital ulcer } \\
\text { disease }\end{array}$ & 1 & $32^{*}$ \\
\hline urethral discharge & 4 & $40^{*}, 41^{*}, 45^{*}, 47^{*}$ \\
\hline \multicolumn{3}{|c|}{ Diseases of the circulatory system } \\
\hline acute cardiac symptoms & 1 & $63^{*}$ \\
\hline \multicolumn{3}{|l|}{ Neoplasms } \\
\hline oral cancer & 3 & $20,43,59$ \\
\hline \multicolumn{3}{|l|}{$\begin{array}{l}\text { Certain infectious or parasitic } \\
\text { diseases }\end{array}$} \\
\hline malaria & 1 & $67^{*}$ \\
\hline tuberculosis & 1 & $80^{*}$ \\
\hline
\end{tabular}




\begin{tabular}{|c|c|c|}
\hline \multicolumn{3}{|l|}{ Product requests } \\
\hline codeine analgesics & 1 & 82 \\
\hline Antibiotics & 1 & $26^{*}$ \\
\hline Criterion & Studies & References \\
\hline \multicolumn{3}{|c|}{$\begin{array}{l}\text { Studies assessing general diagnostic } \\
\text { performance using scenarios of }\end{array}$} \\
\hline Contraceptive management & 1 & 79 \\
\hline \multicolumn{3}{|l|}{$\begin{array}{l}\text { Certain infectious or parasitic } \\
\text { diseases }\end{array}$} \\
\hline vaginal thrush & 2 & 33,34 \\
\hline \multicolumn{3}{|l|}{ Diseases of the visual system } \\
\hline eye discomfort & 1 & 84 \\
\hline \multicolumn{3}{|l|}{$\begin{array}{l}\text { Diseases of the muscoskeletal } \\
\text { system }\end{array}$} \\
\hline back pain & 1 & 84 \\
\hline leg cramps/fatigue & 1 & 24 \\
\hline \multicolumn{3}{|l|}{ Diseases of the nervous system } \\
\hline headache & 4 & $27,31,36,69^{*}$ \\
\hline insomnia & 1 & 55 \\
\hline facial pain & 1 & $56^{*}$ \\
\hline \multicolumn{3}{|c|}{ Diseases of the respiratory system } \\
\hline Acute respiratory infection & 1 & $53^{*}$ \\
\hline allergic rhinitis & 1 & 24 \\
\hline common cold & 6 & $49^{*}, 50,53^{*}, 56^{*}, 79,84,24$ \\
\hline \multicolumn{3}{|c|}{ Diseases of the digestive system } \\
\hline abdominal pain & 1 & 36 \\
\hline indigestion & 1 & 38 \\
\hline vomiting & 1 & 84 \\
\hline dyspepsia & 2 & 48,79 \\
\hline diarrhoea & 4 & $24,69^{*}, 79,84$ \\
\hline Product requests & 11 & $27,31,33,34,38,46,50,53^{*}, 72^{*}, 78,79$ \\
\hline \multicolumn{3}{|l|}{ Study evaluated: } \\
\hline General performance & 22 & $\begin{array}{l}24,27,29-31,33,34,36,38,39^{*}, 46,48,49^{*}, 50,53,55,56^{*}, 69^{*}, \\
72^{*}, 78,79,84\end{array}$ \\
\hline Specific condition & 46 & $\begin{array}{l}20,21^{*}-23,25^{*}, 26^{*}, 28,32^{*}, 35,37,40^{*}, 41^{*}, 42,43,44^{*}, \\
45^{*}, 47^{*}, 51,52,54^{*}, 57,58,59^{*}, 60^{*}, 61^{*}, 62,63^{*}, 64-66 \\
67^{*}, 68^{*}, 70,71^{*}, 73^{*}, 74^{*}, 80^{*}, 83,83^{*}, 85,86^{*}, 87\end{array}$ \\
\hline
\end{tabular}




\begin{tabular}{|c|c|c|c|c|c|c|c|c|c|c|}
\hline $\begin{array}{l}\text { study } \\
\text { reference }\end{array}$ & CR1 & CR2 & CR3 & CR4 & CRTOTAL & MN1 & MN2 & MN3 & MN4 & MNTOTAL \\
\hline 20 & 0 & 0 & 0 & 0 & 0 & 0 & 0 & 0 & 1 & 1 \\
\hline 21 & 1 & 0 & 0 & 0 & 1 & 0 & 1 & 0 & 1 & 2 \\
\hline 22 & 1 & 1 & 0 & 0 & 2 & 1 & 1 & 0 & 1 & 3 \\
\hline 23 & 1 & 0 & 0 & 0 & 1 & 1 & 1 & 0 & 1 & 3 \\
\hline 24 & 1 & 0 & 0 & 1 & 2 & 0 & 1 & 0 & 0 & 1 \\
\hline 25 & 1 & 0 & 0 & 0 & 1 & 0 & 1 & 0 & 1 & 2 \\
\hline 26 & 1 & 0 & 0 & 1 & 2 & 0 & 1 & 0 & 0 & 1 \\
\hline 27 & 0 & 0 & 0 & 0 & 0 & 1 & 1 & 0 & 1 & 3 \\
\hline 28 & 1 & 0 & 0 & 0 & 1 & 1 & 1 & 1 & 1 & 4 \\
\hline 29 & 0 & 0 & 0 & 0 & 0 & 1 & 1 & 1 & 0 & 3 \\
\hline 30 & 0 & 0 & 0 & 0 & 0 & 1 & 1 & 1 & 0 & 3 \\
\hline 31 & 0 & 0 & 0 & 0 & 0 & 1 & 1 & 0 & 1 & 3 \\
\hline 32 & 1 & 0 & 0 & 1 & 2 & 0 & 1 & 1 & 0 & 2 \\
\hline 33 & 1 & 1 & 0 & 1 & 3 & 0 & 1 & 0 & 0 & 1 \\
\hline 34 & 0 & 0 & 0 & 0 & 0 & 1 & 1 & 1 & 0 & 3 \\
\hline 35 & 1 & 0 & 0 & 0 & 1 & 1 & 1 & 0 & 1 & 3 \\
\hline 36 & 1 & 1 & 1 & 1 & 4 & 0 & 0 & 1 & 0 & 1 \\
\hline 37 & 1 & 0 & 0 & 0 & 1 & 1 & 1 & 1 & 1 & 4 \\
\hline 38 & 0 & 0 & 0 & 0 & 0 & 1 & 1 & 1 & 1 & 4 \\
\hline 39 & 0 & 0 & 0 & 0 & 0 & 1 & 1 & 1 & 1 & 4 \\
\hline 40 & 1 & 0 & 0 & 1 & 2 & 0 & 1 & 0 & 0 & 1 \\
\hline 41 & 1 & 0 & 0 & 0 & 1 & 1 & 1 & 0 & 1 & 3 \\
\hline 42 & 1 & 0 & 0 & 0 & 1 & 1 & 1 & 1 & 1 & 4 \\
\hline 43 & 0 & 0 & 0 & 0 & 0 & 0 & 0 & 0 & 1 & 1 \\
\hline 44 & 1 & 0 & 0 & 0 & 1 & 0 & 1 & 1 & 1 & 3 \\
\hline 45 & 1 & 0 & 0 & 0 & 1 & 0 & 1 & 0 & 1 & 2 \\
\hline 46 & 0 & 0 & 0 & 0 & 0 & 1 & 1 & 0 & 1 & 3 \\
\hline 47 & 0 & 0 & 0 & 0 & 0 & 0 & 0 & 0 & 1 & 1 \\
\hline 48 & 1 & 1 & 1 & 1 & 4 & 0 & 0 & 0 & 0 & 0 \\
\hline 49 & 1 & 0 & 0 & 1 & 2 & 1 & 1 & 0 & 0 & 2 \\
\hline 50 & 0 & 0 & 0 & 0 & 0 & 1 & 1 & 0 & 1 & 3 \\
\hline 51 & 1 & 0 & 0 & 1 & 2 & 1 & 1 & 1 & 0 & 3 \\
\hline 52 & 1 & 0 & 0 & 1 & 2 & 1 & 1 & 0 & 0 & 2 \\
\hline 53 & 0 & 0 & 0 & 0 & 0 & 1 & 1 & 0 & 1 & 3 \\
\hline 54 & 0 & 0 & 0 & 0 & 0 & 1 & 1 & 1 & 1 & 4 \\
\hline 55 & 1 & 1 & 1 & 1 & 4 & 0 & 0 & 0 & 0 & 0 \\
\hline 56 & 1 & 0 & 1 & 1 & 3 & 1 & 1 & 0 & 0 & 2 \\
\hline 57 & 1 & 0 & 0 & 0 & 1 & 1 & 0 & 1 & 1 & 3 \\
\hline 58 & 0 & 0 & 0 & 0 & 0 & 1 & 1 & 0 & 1 & 3 \\
\hline 59 & 0 & 0 & 0 & 0 & 0 & 1 & 1 & 1 & 1 & 4 \\
\hline 60 & 0 & 0 & 0 & 0 & 0 & 1 & 1 & 1 & 1 & 4 \\
\hline
\end{tabular}




\begin{tabular}{|c|c|c|c|c|c|c|c|c|c|c|}
\hline 61 & 1 & 0 & 0 & 0 & 1 & 1 & 1 & 1 & 1 & 4 \\
\hline 62 & 1 & 1 & 1 & 1 & 4 & 0 & 0 & 0 & 0 & 0 \\
\hline 63 & 0 & 0 & 0 & 0 & 0 & 1 & 1 & 1 & 1 & 4 \\
\hline 64 & 1 & 0 & 0 & 0 & 1 & 1 & 1 & 0 & 1 & 3 \\
\hline 65 & 1 & 1 & 0 & 1 & 3 & 0 & 1 & 0 & 0 & 1 \\
\hline 66 & 0 & 0 & 0 & 0 & 0 & 1 & 1 & 0 & 1 & 3 \\
\hline 67 & 0 & 0 & 0 & 0 & 0 & 1 & 1 & 1 & 1 & 4 \\
\hline 68 & 0 & 0 & 0 & 0 & 0 & 1 & 1 & 1 & 1 & 4 \\
\hline 69 & 0 & 0 & 0 & 0 & 0 & 1 & 0 & 0 & 1 & 2 \\
\hline 70 & 1 & 1 & 0 & 0 & 2 & 1 & 1 & 0 & 1 & 3 \\
\hline 71 & 1 & 0 & 0 & 1 & 2 & 0 & 1 & 1 & 0 & 2 \\
\hline 72 & 0 & 0 & 0 & 0 & 0 & 1 & 1 & 0 & 1 & 3 \\
\hline 73 & 1 & 0 & 0 & 0 & 1 & 1 & 1 & 0 & 1 & 3 \\
\hline 74 & 0 & 0 & 0 & 0 & 0 & 1 & 1 & 0 & 1 & 3 \\
\hline 75 & 0 & 0 & 0 & 0 & 0 & 1 & 1 & 0 & 1 & 3 \\
\hline 76 & 0 & 0 & 0 & 0 & 0 & 1 & 1 & 1 & 1 & 4 \\
\hline 77 & 1 & 0 & 0 & 0 & 1 & 1 & 1 & 1 & 1 & 4 \\
\hline 78 & 0 & 0 & 0 & 0 & 0 & 1 & 1 & 1 & 1 & 4 \\
\hline 79 & 0 & 0 & 0 & 0 & 0 & 1 & 1 & 0 & 1 & 3 \\
\hline 80 & 0 & 0 & 0 & 0 & 0 & 0 & 0 & 0 & 1 & 1 \\
\hline 81 & 0 & 0 & 0 & 0 & 0 & 1 & 1 & 1 & 1 & 4 \\
\hline 82 & 0 & 0 & 0 & 0 & 0 & 1 & 1 & 1 & 1 & 4 \\
\hline 83 & 0 & 0 & 0 & 0 & 0 & 1 & 1 & 1 & 1 & 4 \\
\hline 84 & 1 & 0 & 0 & 0 & 1 & 1 & 1 & 1 & 1 & 4 \\
\hline 85 & 1 & 0 & 0 & 0 & 1 & 1 & 1 & 1 & 1 & 4 \\
\hline 86 & 1 & 0 & 0 & 1 & 2 & 0 & 1 & 0 & 1 & 2 \\
\hline 87 & 1 & 0 & 0 & 0 & 1 & 0 & 1 & 0 & 1 & 2 \\
\hline MODE & 1 & 0 & 0 & 0 & & 1 & 1 & 0 & 1 & \\
\hline MEDIAN & & & & & 1 & & & & & 3 \\
\hline
\end{tabular}

